\title{
Preface to the special issue on "Clustering and search techniques in large scale networks"
}

\author{
P. M. Pardalos ${ }^{1}$ • V. A. Kalyagin ${ }^{1}$
}

Received: 3 June 2016 / Accepted: 15 June 2016 / Published online: 21 June 2016

(C) Springer-Verlag Berlin Heidelberg 2016

Clustering and search techniques are essential to a wide spectrum of applications. Network clustering techniques are becoming common in the analysis of massive data sets arising in various branches of science, engineering, government and industry. In particular, network clustering and search techniques emerge as an important tool in large-scale networks.

This special issue of Optimization Letters contains refereed selected papers presented at the workshop on clustering and search techniques in large scale networks that took place on November 3-8, 2014 at the Laboratory of Algorithms and Technologies for Networks Analysis (LATNA), Higher School of Economics, in Nizhny Novgorod, Russia. The workshop was supported by the Russian Science Foundation Grant RSF 14-41-00039.

This workshop provided a forum for leading as well as beginning researchers and students to discuss recent advances and identify current and future challenges arising in research concerning clustering and search problems in large networks. The papers of this special issue reflect some the problems discussed at the workshop.

We would like to thank the valuable work of authors and reviewers for making this issue possible.

V. A. Kalyagin

vkalyagin@hse.ru

1 National Research University Higher School of Economics, Nizhny Novgorod, Russian Federation 